\title{
A uvby database for Stephenson-Sanduleak southern luminous stars
}

\author{
B. Cameron Reed \\ Department of Physics, Alma College, Alma, Michigan 48801, U.S.A. \\ e-mail: reed@alma.edu
}

Received September 5; accepted November 13, 1995

\begin{abstract}
A database of published Strömgren four-color uvby photoelectric photometry for objects in the Stephenson-Sanduleak Luminous Stars in the Southern Milky Way catalog has been compiled from the literature. Of 5132 stars in the catalog, 817 have uvby colors and magnitudes available; altogether, the database incorporates 1181 observations from 41 references. The database is available electronically at CDS, and lists LS number, HD or CD number, $b-y, m_{1}, c_{1}$, number of observations, numeric codes for notes and references, galactic $\ell$ and $b$ coordinates, and aliases for named or variable stars.
\end{abstract}

Key words: stars: early-type — stars: fundamental parameters — stars: general — catalogs

\section{Introduction}

This paper is the third in a series devoted to compiling fundamental observational data for stars listed in Stephenson \& Sanduleak's (1971) catalog of Luminous Stars in the Southern Milky Way (LS stars). Constituting as it does a fairly complete guide to over 5100 intrinsically luminous stars (mostly OB stars and A and F supergiants) within 10 degrees of the southern galactic plane to a limiting magnitude of about 12.5, the LS catalog has served for a quarter- century as a starting point for numerous studies of southern Milky Way star clusters, dynamics, and reddening. Paper I of the present series (Reed 1993) compiled $U B V \beta$ photometry for these objects and Paper II (Reed \& Beatty 1995) MK-system spectral classifications; these databases have continued to grow since those papers appeared, and, at this writing, incorporate over 9300 and 6400 observations, respectively. Full $U B V$ data are now available for nearly 3400 stars in addition to 220 stars for which $\beta$ values are available but which lack $U B V$, and nearly 2600 stars have MK classifications available. The present paper compiles published Strömgren fourcolor uvby photometry of the LS stars in the form of $b-y$ colors, the metallicity index $m_{1}=(v-b)-(b-y)$, and the Balmer discontinuity index $c_{1}=(u-v)-(v-y)$.

\section{Strategy}

The general precepts used in compiling the $U B V \beta$ and MK databases for the LS stars are retained here: only original data from published sources are incorporated. Data from individual references are maintained as separate com- puter files, then the individual files are collated together to produce an overall compilation.

A two-stage process was followed in assembling this database. First, all references from Papers I and II which also contained original uvby photometry were isolated; this proved to be 24 papers reporting some 800 observations of 700 individual stars. Second, the SIMBAD database was queried for availability of $u v b y$ data for each LS star and notes made as to references to be acquired and reviewed for suitability. It is worth emphasizing that by "original" data is meant only that which could with reasonable certainty be determined as being original to a given reference. Observations attributed by source A to source B, for example, appear here credited only to source $\mathrm{B}$ and are not repeated under source A. Particular care was taken to exclude any results adopted from or that derived from averaging or homogenizing data from different sources. [Eventually, it is planned to reduce the data to a common system along the lines followed by Hauck \& Mermilliod (1990)]. As in Papers I and II, each reference was examined for any relevant notes (emission, variability, duplicity, etc.), which are recorded in the database via numeric codes. As remarked in paper I, a chronic problem area is the number of measurements reported for each star: while most authors state this explicitly, some give only a minimum number and others no details at all. If no information along these lines was evident, the number of measurements was set by default to be one.

The present database does not incorporate $V$ magnitudes derived from the Strömgren photometry; Johnsonsystem $V$ magnitudes are available in Paper I. 


\section{The database}

The final database is available electronically from CDS, and consists of 1181 records, one for each published observation of an LS star. Each record consists of 12 columns: (1) LS number, (2) notes flag, (3) HD or CD number, (4)-(6) $b-y, m_{1}, c_{1}$, (7) number of measurements, (8) numerical note and (9) reference codes (see Table 1 below), (10)-(11) galactic $\ell$ and $b$ coordinates, and (12) variable name or HR number where appropriate. An asterisk in Col. (2) indicates that a note concerning that star appears in Sect. 4 below. For stars with multiple entries, the LS and $\mathrm{HD} / \mathrm{CD}$ numbers as well as $\ell$ and $b$ and variable names are repeated in subsequent records. Beyond attempting to resolve apparent typographical errors, misidentifications, and situations where data were clearly discordant, no attempt has been made at critically assessing the compiled data. While a careful effort at proofreading and resolving conflicts was made, errors and omissions no doubt remain, and the author would welcome being informed of any that users discover.

Note codes (Col. 8) are identical to those used in Paper I with two exceptions: note 12 refers to uvby photometry, and three new notes (Nos. 20-22) have been added; the complete set of codes is given here for convenience:

- 1. Emission

- 2. Double or Spectroscopic Binary

-3 . Variable or suspected variable

- 4. Subdwarf

- 5. P Cygni star

-6 . Wolf-Rayet star

-7 . See comments in reference cited

$-8 . \beta$ uncertain

-9 . Identification uncertain

- 10. $\beta$ quoted to two decimal places only

-11 . Of star

- 12. uvby uncertain

- 13. Combined result taken from reference cited

- 14. Crowded field or faint companion(s)

-15 . Brighter star of a pair with same LS number quoted in reference cited

- 16. Radial velocity variable

- 17. Double-line spectroscopic binary

- 18. Combined photoelectric/photographic observation

- 19. Data deriving from CCD observations

- 20. Carbon star

-21 . $\beta$ Cephei variable

- 22. uvby reported to two decimal places only

Since some of these codes are relevant only to Paper I, they will not appear in the present database; conversely, more than one may apply for some stars, a situation which necessitated judgments as to which was the most serious. In any event, users are encouraged to treat this compilation as a sourcebook and to consult Papers I and II and the original literature for any further information in any particular case.
Table 1. References cited and compilation

\begin{tabular}{|c|c|}
\hline Number & Reference \\
\hline $\begin{array}{l}1 \\
2 \\
3 \\
4 \\
5 \\
6 \\
7 \\
8 \\
9 \\
10\end{array}$ & $\begin{array}{l}\text { Crawford, Barnes, \& Golson (1970) } \\
\text { Perry (1991) } \\
\text { Crawford, Barnes, \& Golson (1971a) } \\
\text { Knude (1977) } \\
\text { Loden (1979) } \\
\text { Perry, et. al. (1976) } \\
\text { Crawford, et. al. (1971) } \\
\text { Lynga (1977) } \\
\text { Eggen (1977a) } \\
\text { Stetson (1981) }\end{array}$ \\
\hline $\begin{array}{l}11 \\
12 \\
13 \\
14 \\
15 \\
16 \\
17 \\
18 \\
19 \\
20\end{array}$ & $\begin{array}{l}\text { Knude (1992) } \\
\text { Crawford (1975) } \\
\text { Crawford, Barnes, \& Golson (1971b) } \\
\text { Kaltcheva \& Georgiev (1992) } \\
\text { Gray \& Olsen (1991) } \\
\text { Kaltcheva \& Georgiev (1993) } \\
\text { Franco (1994) } \\
\text { Terranegra, et. al. (1994) } \\
\text { Kaltcheva \& Georgiev (1994) } \\
\text { Shobbrook \& Lynga (1994) }\end{array}$ \\
\hline $\begin{array}{l}21 \\
22 \\
23 \\
24 \\
25 \\
26 \\
27 \\
28 \\
29 \\
30\end{array}$ & $\begin{array}{l}\text { Kaltcheva \& Georgiev (1995) } \\
\text { Shobbrook (1983a) } \\
\text { Shobbrook (1983b) } \\
\text { Shobbrook (1984) } \\
\text { Eggen (1978) } \\
\text { Eggen (1977b) } \\
\text { Shaw (1975) } \\
\text { Kilkenny (1978) } \\
\text { Gronbech \& Olsen (1976) } \\
\text { Danziger \& Faber (1972) }\end{array}$ \\
\hline $\begin{array}{l}31 \\
32 \\
33 \\
34 \\
35 \\
36 \\
37 \\
38 \\
39 \\
40 \\
41\end{array}$ & $\begin{array}{l}\text { Stokes (1972) } \\
\text { Eggen (1981) } \\
\text { Brandt, et. al. (1971) } \\
\text { Morrison (1975) } \\
\text { Kilambi (1975) } \\
\text { Heck \& Manfroid (1975) } \\
\text { Hesser (1976) } \\
\text { Hartwick \& Hesser (1973) } \\
\text { Heck (1977) } \\
\text { Balona \& Koen (1994) } \\
\text { Corradi \& Franco (1995) }\end{array}$ \\
\hline
\end{tabular}

\section{Remarks on individual stars}

Thirteen stars in the database are flagged with asterisks in Col. (2); notes for these are given here. To save space, only important notes or those that are new since the publication of Paper I are given here. Many of the stars in the database have notes relevant to their $U B V \beta$ photometry; the present database should be used in conjunction with Paper I. Each note begins with an LS number in boldface followed by parentheses containing the photographic magnitude of the star as given in the LS catalog.

98 (7.5) Variations in the spectrum of this Wolf-Rayet star are discussed by Wilson, 1948, PASP 60, 383, and a fluxcalibrated spectrum of this star to the Lyman limit is discussed in Mccandliss, et al., 1993, ApJ 416, 372. Howarth 
\& Schmutz, 1995, A\&A 294, 529, report a distance of $1.8 \mathrm{kpc}$ for this star.

166 (7.4) Gies, et al., 1994, ApJ 422, 823, remark that this star is a spectroscopic binary with a period of 6.0173 days. The companion has a mass ratio $0.23 \pm 0.04$, and a magnitude difference $3.3+0.4$.

949 (2.0) Reported as variable by Balona, 1992, MNRAS 254, 404-412.

1657 (8.3) See notes in Paper I.

1673 (9.1) In NGC 3293. Stephenson and Sanduleak indicate this star as number 21 in the finding chart of Feast, 1958, MNRAS 118, 618. However, references 30 and 31 in Paper I both indicate that this star is an M1 supergiant with $B-V=2.0$. When precessed to 1875 , the coordinates of LS 1673 match very closely to those of CPD -57 3506 , a B1 II star which is number 22 in Feast's chart. I have assumed that Stephenson and Sanduleak marked the wrong star; the data given is that for Feast's number 22. 1679 (10.0) See notes in Paper I.

1688 (8.8) See notes in Paper I.

1689 (8.0) HD 92025. Misidentified in the LS catalog as HD 92007

2212 (7.7) As LS 949.

2803 (6.7) uvby colors given by Crawford et al. (1971a; Ref. 3 in Table 1) for HR 4890 (= LS 2813) correspond to those of LS 2803 according to other sources; their data have been attributed to LS $2803=$ HR 4887. In NGC 4755.

2813 (6.7) See comments for 2803.

3231 (6.0) HX Lup.

3331 (5.0) Delta Circinus.

Overall, the database incorporates 1181 observations (defined as single or multiple measurements of a given star) on 817 stars from 41 references; the number of observations per star varies from one (545 stars) to seven (one star, LS 2803). Clearly, much fundamental data remains to be acquired for the LS stars: for the most part, the stars that appear in the present database are those from the brighter end of the LS magnitude distribution $\left(m_{\mathrm{pg}}<10\right)$. Presently, only some 600 stars have both $U B V \beta$ and Strömgren photometry available as well as MK-system spectral classifications. An up-to-date compilation of all data for the LS stars is available from the author, either as a printout (approx. 180 pages), by e-mail, or in the form of three separate, collated files on 3.5-inch Macintosh-formatted diskettes.

Acknowledgements. It is a pleasure to thank the Inter-Library loan staff at Alma College for efficiently tracking down a number of references, and Dr. Carme Jordi for a number of useful remarks. This research has made use of the Simbad database, operated at CDS, Strasbourg, France.

\section{References}

Balona L.A., Koen C., 1994, MNRAS 267, 1071

Brandt J.C., Stecher T.P., Crawford D.L., Maran S.P., 1971, ApJ 163, L99

Corradi W.J.B., Franco G.A.P., 1995, A\&AS 112, 95

Crawford D.L., Barnes J.V., Golson J.C., 1970, AJ 75, 624

Crawford D.L., Barnes J.V., Hill G., Perry C.L., 1971, AJ 76, 1048

Crawford D.L., Barnes J.V., Golson J.C., 1971a, AJ 76, 621

Crawford D.L., Barnes J.V., Golson J.C., 1971b, AJ 76, 1058

Crawford D.L., 1975, PASP 87, 481

Danziger I.J., Faber S.M., 1972, A\&A 18, 428

Eggen O.J., 1977a, PASP 89, 205

Eggen O.J., 1977b, ApJS 34, 1

Eggen O.J., 1978, PASP 90, 436

Eggen O.J., 1981, ApJ 247, 507

Franco G.A.P., 1994, A\&AS 104, 9-22

Gray R.O., Olsen E.H., 1991, A\&AS 87, 541

Gronbech B., Olsen E.H., 1976, A\&AS 25, 213

Hartwick F.D.A., Hesser J.E., 1973, ApJ 186, 1171

Hauck B., Mermilliod J.C., 1990, A\&AS 86, 107

Heck A., Manfroid J., 1975, A\&AS 22, 323

Heck A., 1977, A\&AS 27, 47

Hesser J.E., 1976, PASP 88, 849

Kaltcheva N.T., Georgiev L.N., 1992, MNRAS 259, 166

Kaltcheva N.T., Georgiev L.N., 1993, MNRAS 261, 847

Kaltcheva N.T., Georgiev L.N., 1994, MNRAS 269, 289

Kaltcheva N.T., Georgiev L.N., 1995 (Private Communication)

Kilambi G.C., 1975, PASP 87, 975

Kilkenny D., 1978, MNRAS 182, 629

Knude J.K., 1977, A\&AS 30, 297

Knude J.K., 1992, A\&AS 92, 841

Loden L.O., 1979, A\&AS 36, 83

Lynga G., 1977, A\&A 54, 71

Morrison N.D., 1975, ApJ 200, 113

Perry C.L., Franklin C.B., Landolt A.U., Crawford D.L., 1976, AJ 81, 632

Perry C.L., 1991, PASP 103, 494

Reed B.C., 1993, ApJS 87, 367

Reed B.C., Beatty A.E., 1995, ApJS 97, 189

Shaw J.S., 1975, A\&A 41, 367

Shobbrook R.R., 1983a, MNRAS 205, 1215

Shobbrook R.R., 1983b, MNRAS 205, 1229

Shobbrook R.R., 1984, MNRAS 206, 273

Shobbrook R.R., Lynga G., 1994, MNRAS 269, 857

Stephenson C.B., Sanduleak N., 1971, Publ. Warner Swasey Obs. 1, 1

Stetson P.B., 1981, AJ 86, 1500

Stokes N.R., 1972, MNRAS 160, 155

Terranegra L., Chavarria-K C., Diaz S., Gonzalez-Patino D., 1994, A\&AS 104, 557 